\title{
The network interplay of interferon and toll-like receptor signaling pathways in the anti-Candida immune response
}

\section{Ranieri Coelho Salgado ( $\square$ ranierics@usp.br)}

Universidade de São Paulo

Dennyson Leandro M. Fonseca

Universidade de São Paulo

Alexandre H. C. Marques

Universidade de São Paulo

Sarah M. S. Napoleao

Universidade de São Paulo

Tabata Takahashi Franca

Universidade de São Paulo

Karen Tiemi Akashi

Universidade de São Paulo

Caroline A. S. Prado

Universidade de São Paulo

Gabriela Crispim Baiocchi

Universidade de São Paulo

Desiree R. Placa

Universidade de São Paulo

Gabriel Jansen-Marques

Universidade de São Paulo

Igor Salerno Filgueiras

Universidade de São Paulo

Roberta De Vito

Brown University

Paula Paccielli Freire

Universidade de São Paulo

Gustavo Cabral de Miranda

Universidade de São Paulo

Niels Olsen Saraiva Camara Universidade de São Paulo

Vera L. G. Calich 
Universidade de São Paulo

Hans D. Ochs

University of Washington

Lena F. Schimke

Universidade de São Paulo

Igor Jurisica

University of Toronto

Antonio Condino-Neto

Universidade de São Paulo

Otavio Cabral-Marques

Universidade de São Paulo

\section{Research Article}

Keywords: Candida spp., network interplay, toll-like receptor (TLR), interferon (IFN) signaling, immune response

Posted Date: September 13th, 2021

DOI: https://doi.org/10.21203/rs.3.rs-279551/v3

License: (c) (1) This work is licensed under a Creative Commons Attribution 4.0 International License. Read Full License 


\section{Abstract}

Fungal infections represent a major global health problem that affects over a billion people and kills more than 1.5 million individuals annually. Here we employed an integrative approach to unravel the landscape of the human immune responses to Candida spp. by performing a meta-analysis of microarray, bulk, and single-cell RNA-sequencing (RNASeq) of blood transcriptome data. We identified across these different studies a consistent interconnected network interplay of signaling molecules involved in both toll-like receptor (TLR) and interferon (IFN) signaling cascades that is activated in response to different Candida species (C. albicans, C. auris, C. glabrata, C. parapsilosis, and C. tropicalis). Among these molecules, there are several types I IFN, indicating an overlap with the anti-viral immune responses. scRNAseq data confirmed that genes commonly identified by the three transcriptomic methods present a cell-type specific expression patterns across innate and adaptive immune cells. Thus, these data shed new lights on the anti-candida immune response, providing putative molecular pathways for therapeutic intervention.

\section{Introduction}

Fungal infections, including the emergence of new fungal pathogens highly resistant to antifungal drugs, represent a major global health issue ${ }^{1-5}$. Fungal infections affect over a billion people worldwide and kill more than 1.5 million individuals annually. Among them, invasive candidiasis (IC) is the most common fungal disease, affecting approximately 250,000 people annually and causing more than 50,000 deaths ${ }^{6,7}$. The increasing number of patients with malignancies, inborn errors of immunity (IEI), autoimmune diseases (receiving immunosuppressive treatment), and hematopoietic stem cell or organ transplant recipients contributes to this high frequency of individuals susceptible to these life-threatening fungal pathogens $s^{8,9}$. Thus, demanding a better understanding of molecular pathways that can be further explored to develop new therapies to reduce morbidity and mortality caused by Candida infections ${ }^{10,11}$.

Linear and mechanistic approaches have elegantly demonstrated that the anti-fungal immune response involves the appropriate recognition of pathogen-associated molecular patterns (PAMPs) by different pattern recognition receptors (PRRs) expressed on the cell membrane such as C-type lectin receptors (CLRs: dectin-1, dectin-2, and CD209), scavenger receptors (CD36), and toll-like receptors (TLRs), e.g., TLR2 and 4. Intracellular PRRs including RIG--llike receptors (RLRs: melanoma differentiation-associated protein 5 or MDA5), TLRs (e.g., TLR3 and TLR9), and NOD-like receptors (NLRs: nucleotide-binding oligomerization domain-containing protein or NOD1/2, NOD-, LRR- and pyrin domain-containing 3 or NLRP3) are also relevant and expressed by antigen-presenting cells and phagocytes, which bind to wellknown ligands ${ }^{12-14}$. Activation of PRRs induces several signaling events such as the canonical Nuclear factor (NF)-KB pathway ${ }^{15}$ that trigger effector anti-fungal mechanisms such as phagocytosis, production of reactive oxygen species (ROS) ${ }^{16}$, degranulation, and neutrophil extracellular traps (NETs) ${ }^{17,18}$. Simultaneously, PRRs promote the production of key inflammatory cytokines such as tumor necrosis 
factor (TNF)-a, Interleukin (IL)-1 $\beta$, IL-6, IL-17, type I Interferons (IFNs [IFN- $\alpha / \beta]$ ), and the IL-12/IFN- $\gamma$ $\operatorname{axis}^{11,14,19,20}$, which shape and instruct immune cells ${ }^{14}$.

However, the landscape of anti-fungal molecules in a holistic and integrative way remains to be provided. To reach this goal, we performed a meta-analysis of blood transcriptome data of microarray, bulk, and single-cell RNA-sequencing (scRNAseq) to unravel the landscape of the human immune responses to Candida spp.. This integrative approach revealed a previous unnoted network interplay of type 1 interferon and toll-like receptor signaling in the anti-candida immune response.

\section{Results}

Multi-layered conservation of TLR and IFN signaling pathways in response to $C$. albicans. We surveyed published RNAseq datasets and found a total of 8 datasets related to the human immune response to Candida spp., being 5 of microarray, 2 bulk RNAseq, and one scRNAseq (further details in the Methods section). First, we explored the scRNAseq by performing over representation analysis (ORA) of differentially expressed genes (DEGs) from innate immune (monocytes, natural killer, and plasmacytoid dendritic cells) and adaptive cells (CD4+, CD8+, and CD19+ lymphocytes), which were assigned to clusters as previously described ${ }^{21}$ (Fig. 1a) in resting and C. albicans conditions (Fig. 1b-C). A total of 6722 DEGs (Suppl. Table S1) were present in these clusters when comparing C. albicans-activated to resting cells. Enriched pathways associated with the immune response to $C$. albicans are shown in Fig. 1d while all enriched categories are present in Suppl. Table S2. Among them, there are 72 and 99 DEGs belonging to TLR and IFN (both type I and type II) signaling cascades. Among them, 62 DEGs are involved in both TLR and IFN signaling cascades based on our enrichment analysis or as previously reported in the literature (Suppl. Table S3).

We next asked whether the interplay between TLR and IFN signaling cascades is also induced at a topological level. To address this issue, performed modular gene co-expression analysis ${ }^{22}$, using the microarray dataset from Smeekens et $\mathrm{al}^{11}$. This is the unique public dataset available containing more than 15 samples per group (30 resting and $24 \mathrm{C}$. albicans-activated samples), required to obtain biologically meaningful modular networks ${ }^{23}$. Modular gene co-expression analysis using CEMiTool ${ }^{24}$ identified thirteen enriched co-expression modules from the total expressed genes by PBMCs (which contain lymphocyte subpopulations, monocytes, and dendritic cells). Among these modules, 12 were significantly enriched (9 downregulated and 3 upregulated) in response to $C$. albicans infection (Fig. 1e). Of note, modules M1 and M2 indicate gene co-expression and upregulation of IFN and interleukin signaling with TLR cascades (Fig. 1f-i).

Based on the results obtained by the modular co-expression analysis, we dissected the significantly enriched pathways from differentially expressed genes (DEGs) induced by $C$. albicans ${ }^{11}$. In agreement with the topological results obtained using CEMiTool, ORA of DEGs using the ClusterProfiler tool ${ }^{25}$ pinpointed different clusters related to the activation of the TLR and IFN signaling cascades (Suppl. Fig. 1a-b). The relationship between the 30 most enriched pathways and their associated genes is shown in a 
network view (Suppl. Fig. 1c) while the entire list of all enriched pathways is summarized in Suppl. Table S4. Type I IFN signaling was the most significant pathway modulated by $C$. albicans, as previously reported by Smeekens et al. ${ }^{11}$ and as recently characterized by Bruno et al. $(2021)^{26}$. Furthermore, $C$. albicans activation significantly enriched several TLR signaling events such as TLR4, TLR3, TLR7/8, and TLR9 as well as MyD88/TIR-domain-containing adapter-inducing interferon- $\beta$ (TRIF)/TIR Domain Containing Adaptor Protein (TIRAP) cascades, and TRAF6-mediated NF-KB activation. ORA also indicated that $C$. albicans activates chemokine (G protein-coupled receptors [GPCR] ligand binding) and cytokine signaling pathways (IL-10, IL-3 and IL4), IFN- $\alpha / \beta$ signaling, Interferon-stimulated gene 15 (ISG15) antiviral mechanism, TNF Receptor Associated Factor 3 (TRAF3)-dependent IRF activation, DExD/H-Box Helicase 58 (DDX58)/Interferon Induced with Helicase C Domain 1 (IFIH1)-mediated induction of IFN- $\alpha / \beta$, and regulation of type I and II IFN among the IFN signaling events (Suppl. Fig 1a and c; Suppl. Table S4). This observation agrees with the studies performed by Jaeger et al. ${ }^{27}$, which characterized in detail the relevance of IFIH1 (MDA5) in the anti-candida immune response.

\section{C. albicans infection activates common TLR- and IFN-associated genes in peripheral blood leukocytes}

We further investigated which DEGs and signaling pathways are consistently activated by $C$. albicans in peripheral blood leukocytes such as PBMCs (studies from Smeekens et al. ${ }^{11}$ and Bruno et al. ${ }^{28}$ ) and peripheral whole blood cells (WBCs, studies from Dix et al. ${ }^{29}$ and Sieber \& Kämmer et al. ${ }^{30,31}$ ) throughout all publicly available datasets. WBCs contain PBMCs (lymphocytes $20-45 \%$ and monocytes $2-10 \%$ ) and granulocytes (neutrophils: 50-70\%; basophils: 0-1\%; and eosinophils: 1-5\%) ${ }^{32}$. A meta-analysis of WBCs and PBMCs gene expression datasets using the P-value combination method revealed 44 commonly activated DEGs (40 upregulated and 4 downregulated) (Fig 2a, Suppl. Table S5). According to the cell population, these DEGs form well-defined hierarchical clusters, i.e., PBMCs datasets present a closer expression pattern among them and WBCs datasets, when we compare both regulation and significance (Fig 2b). Enrichment analyses using EnrichR of these 44 genes revealed 87 significantly affected pathways (Suppl. Table S6), including TLR and IFN- $\alpha / \beta$ signaling pathways (Fig. $2 \mathrm{c}$ ). Furthermore, these 44 DEGs also enrich other related interleukin signaling pathways such as JAK-STAT, IL-12, IL-17, IL-23, TNF, and chemokines (GPCR ligand binding) and PRRs, including RIG-I like receptor and NOD signaling. Multi-study Factor Analysis of eligible ${ }^{33}$ datasets (WBCs: Dix et al. ${ }^{29}$ and PBMCs: Smeekens et al. ${ }^{11}$ ); those with the minimal number of samples required for this analysis) identified two common latent factors with high loadings, while specific latent factors showed low loadings across these studies. Thus, strengthening the biological relevance of these 44 common genes (Fig. 2d, Suppl. Table S7).

\section{C. albicans activates common TLR and IFN signaling pathways across different layers of immunity}

Subsequently, we added monocyte-derived dendritic cells (moDCs) studies (Dix et al., Rizzetto et al., and Rizzetto et al. $)^{34-36}$ into our integrative analysis. moDCs are known to be essential players of anti-fungal immunity, bridging the immune system's innate and adaptive arms. We searched for genes commonly regulated by $C$. albicans in transcriptomes of WBCs, PBMCs, and moDCs, in resting or $C$. albicans activation conditions. Intersection analyses performed according to cell population identified 123,223 , 
and 57 common DEGs among WBCs, PBMCs, and moDCs datasets, respectively (Fig. 3a-c). Except for 5 genes (RIN2, RGL1, MARCKS, FNIP2, and TLR7) commonly differentially expressed among the PBMC studies, which were upregulated in the dataset from Smeekens et al. ${ }^{11}$ and downregulated in the Bruno et al. ${ }^{28}$ dataset, all other common DEGs were consistently up- or down-regulated across the studies investigating the same cell population (Suppl. Table S9).

However, only 2 common DEGs were present across all seven datasets (Fig. 3d), which by themselves do not significantly enrich signaling pathways. We then asked if DEGs from each dataset enrich common signaling biological processes among all studies. Gene Ontology (GO) analysis using ClusterProfiler analysis revealed 173 common biological processes (Suppl. Table S8). We found several molecules/pathways essential for the anti-fungal immune response ${ }^{12}$. Among them is a cluster of IFN-y, and NF-kB signaling, and a previously described overlap with the immune response to virus ${ }^{11}$ (Fig. $3 e$ ). This latter has been mechanistically characterized by Bourgeois et al. ${ }^{37}$. Additional ORA of DEGs involved in this cluster found significant enrichment of signaling cascades of single TLRs (TLR2, TLR3, TLR4, TLR5, TLR9, TLR9, and TLR10), TLR heterodimers (TLR1/TLR2, TLR2/TLR6, TLR7/8), and TLR adapter molecules (MyD88/TIRAP, TRAF6, TRIF) as well as several interleukin signaling pathways such as IL-1, IL4/IL-13, IL-6, IL-10, IL-17, and IFN-a/ $\beta$ (Fig. 3f). We found 1096 DEGs (Suppl. Table S11) affecting common biological processes among WBCs, PBMCs and moDCs. Fig. $\mathbf{3 g}$ shows the interactome obtained from some of these 1096 DEGs and enriched signaling cascades (Suppl. Table S12), thus highlighting the association of TLR- and IFN-signaling cascades that were consistently enriched during our analyses. These 1096 DEGs also enrich other PRR and Interleukin signaling pathways such as CLRs (dectin-1), NLRs (NOD1/2), pro- (IL-1, IL6, IL-17, IL-12), anti-inflammatory (IL-10), and T helper 2 (IL-4 and IL-13) cytokines. This immunological balance between a pro-and anti-inflammatory event is crucial for properly controlling fungal infections while maintaining immune homeostasis ${ }^{38,39}$.

\section{C. albicans infection increases the correlation between TLR- and IFN-associated genes}

After verifying TLR and type I and II IFN signaling cascades' consistency, we assessed the degree of association between these two variables during the immune response to $C$. albicans. Due to the minimum sample size requirement ${ }^{40}$, we selected TLR and IFN-associated genes present in the PBMCs transcriptome data from Smeekens et al. ${ }^{11}$. This dataset contains 45 and 14 TLR- and IFN-associated DEGs modulated by $C$. albicans compared to the resting group. $C$. albicans infection increased mainly positive correlations between TLR- and IFN-associated DEGs (Fig. 4a-b). We performed Canonical Correlation Analysis (CCA) to further assess the association's strength between TLR and IFN DEGs. CCA is a generic parametric model used to quantify relationships between two groups of interrelated and interdependent variables ${ }^{41}$. This approach unveiled a pair of canonical variates (x-CV1 and y-CV1), highlighting the strong association between most of TLR - and IFN -associated DEGs in both resting and C. albicans-infected PBMCs (Fig. 4c), although they are able to stratify these conditions (Fig. 4d-e). 
The interplay between TLR and IFN signaling pathways is conserved in response to non-albicans candida species

We asked if only $C$. albicans induces the association between TLR- and IFN-associated genes or other non-albicans candida species, such as $C$. glabrata, C. parapsilosis, $C$. tropicalis, and also the multidrugresistant $C$. auris ${ }^{28,42}$. To address this issue, we used the public dataset from Sieber \& Kämmer et al. ${ }^{30,31}$. Intersection analyses performed with TLR- and IFN-associated DEGs from this dataset revealed 12 and 19 common DEGs, respectively, upon activation by the different Candida species. Furthermore, these DEGs are involved in several pathways related to both TLR and IFN signaling pathways (Fig. 5a-b, Suppl. Table S13).

We also asked whether this interplay between TLR and IFN signaling pathways is also involved in response to the multidrug-resistant $C$. auris. Thus, we explored the unique publicly available dataset analyzing the immune response to $C$. auris and $C$. albicans (Bruno et al. ${ }^{28}$ ). Similar to $C$. albicans activation, ORA of DEGs induced by $C$. auris included TLR and IFN signaling cascades among the 30 most enriched pathways (Suppl. Fig. 2a-b). C. albicans and C. auris similarly modulated DEGs' levels involved in TLR signaling, including NF-KB1, NF-KB2, JUN, and DUSP4, as well as IFN signaling such as IRFs, GBPs, SOCS1, ISG20, TRIM, and IFIT3 (Fig. 5c). When we compared the DEGs induced by $C$. auris with those enriching common pathways among all datasets (1096 DEGs, Suppl. Table S11) assessing the immune response to $C$. albicans, we identified 237 common DEGs (Fig. 5d). ORA of these common DEGs indicates that the interplay between TLR and IFN signaling cascades is a consistent immunologic feature in response to these two Candida species (Fig. 5e).

\section{Inborn errors of immunity (IEI) corroborate the interplay between TLR and IFN signaling cascades}

Finally, we aimed to evaluate the potential clinical and translational relevance of the TLR- and IFNassociated genes and molecular pathways consistently modulated by Candida. Therefore, we searched for IEl associated genes that are known to increase human susceptibility to both systemic and mucocutaneous candidiasis as described by Tangye et al. ${ }^{43}$. For this analysis we only include mutations, but not single-nucleotide polymorphisms (SNPs) associated with susceptibility to candida infections. Hence, despite Jaeger et al. ${ }^{27}$ have characterized that SNPs in MDA5 are associated with candida, mutation in this gene have been so far reported as an inborn error of immunity associated with increased susceptibility to recurrent viral infections ${ }^{43}$. Nonetheless, this fact reinforces type I interferon's consistent role in the anti-candida immune response, indicating that patients with increase susceptibility to Candida spp. need to be screened for mutations in MDA5.

So far, mutations in 100 genes known to beassociated with IEl have been identified to increased susceptibility to candidiasis and often other clinical manifestations (Suppl. Table S16). We compared them with the 1096 genes (Suppl. Table S11, i.e., those enriching the common biological processes activated by C. albicans (Fig. 3e). These 1096 genes encode molecules present in different compartments such as extracellular regions, organelles, and nuclei, forming macromolecular complexes. Together, they 
form a highly interconnected physical protein-protein interaction network (Fig. 6a), which contains several hubs $^{44}$ (Fig. 6b), defined as those having more than or equal to 200 interaction partners. Of note, 34 genes associated with IEI are also present across the studies. Meanwhile, although 66 genes associated with IEl are not identified in the datasets, these genes are highly connected with the other DEGs in this network. Furthermore, the 1096 DEGs mostly contain Type I and II IFN- associated genes, 868 in total (Fig. 6c, Suppl. Table S14).

The 34 IEl associated genes present in this network underly 7 groups of IEl including congenital defects of phagocytes, defects of intrinsic and innate immunity, predominantly antibody deficiencies, and diseases of immune dysregulation, as defined by the International Union of Immunological Societies Expert Committee(IUIS) ${ }^{43}$ (Suppl. Fig. 3a). Notably, among the hubs are STAT1, STAT3, NFKBIA (IKBa), and NFKB1, which are well known to be associated with TLR and IFN signaling pathways ${ }^{45-50}$. ORA of these 34 genes indicates that in addition to dectin- $1^{51}$ and NLRs signaling, they mostly enrich both type I and II IFN and several TLR signaling pathways (TLR1/2, TLR2/6, MyD88, and TRAF6-mediated NF-KB activation) (Suppl. Fig. 3b-c).

\section{Common TLR-and IFN-associated DEGs and signaling pathways across microarray, bulk, and single-cell RNA-seq datasets}

Finally, we revisited the scRNAseq data and found that 11 TLR- and 23 IFN-associated DEGs are also among those WBCs, PBMCs and moDCs DEGs identified by microarray and bulk RNAseq datasets (Suppl. Table S15). Thus, indicating that the network interplay of TLR- and IFN-associated DEGs are not a particular feature of a specific leukocyte cell population since $C$. albicans systemically activated this network throughout the different innate (monocytes, natural killer, and plasmacytoid dendritic cells) and adaptive cells (CD4+, CD8+, and CD19+ lymphocytes) identified by the scRNAseq dataset. Fig. 7a-b illustrate these 34 common genes across the leukocytes subpopulations and those present in the WBCs, PBMCs, and moDCs datasets (Fig. 2a-c). Hierarchical clustering of common enriched pathways across the cell subpopulations identified by scRNAseq showed a similar up-regulation pattern of TLR- and IFNassociated signaling pathways, forming clusters (Fig. 7c), as seen by microarray and bulk RNAseq. Taken together, these data strongly support the immunobiological relevance of the interplay between TLR and IFN signaling cascades, as previously described in the literature ${ }^{50,103-109}$ (Fig. 8).

\section{Discussion}

The association between PRR activation and cytokine production by immune cells is crucial for an adequate immune response against pathogens and has been abundantly investigated by linear approaches or strategies designed to identify the anti-fungal transcriptomic signature ${ }^{11,28,52,53}$. For instance, several immunologic molecules and pathways such as those triggered by TLR and IFN, which induce the generation of T cell subpopulations (e.g., T helper 1 [Th1], Th17, and T regulatory [T reg] cells) have been successfully characterized by individual studies and mechanistic approaches ${ }^{14}$. Our systems immunology approach integrates dispersed transcriptomic studies that investigated the anti-candida 
immune response, highlighting the consistency of the crosstalk between PRRs (e.g., CLRs, TLRs, and NLRs) and type I and type II interferons as well as cytokine (e.g., TNF, and IL-10) cascades previously reported $^{37,54-60}$. Besides, we show a consistent overlap between the antiviral and antifungal immune responses, which supports the previously reported pivotal role of IFN type I in the immune response against $C$. albicans ${ }^{11}$.

Of note, recent reports suggest that the Candida-specific induction of type I IFN responses is not only limited to professional immune cells since in vivo studies confirm the role of type I IFN in epithelial host defense against $C$. albicans ${ }^{61,62}$. In agreement, a transcriptional profiling study shows that the type I IFN response is also induced by vaginal epithelial cells in response to a variety of candida species ${ }^{63}$. However, while the type I IFN response showed a protective role at the early stages of infection, opposite effects (tissue damage) were observed at the later stages. This aligns with several studies showing that IFNs may play beneficial or detrimental roles in the host defense against candidiasis. I.e., while these cytokines are essential to control the development of severe candida infections by promoting the inflammatory action of phagocytic cells (monocytes and neutrophils), the uncontrol activation of these cells by IFNs during sepsis may contribute to fatal tissue damage $\mathrm{e}^{64-67}$. Therefore, understanding the TLR-IFN network during different timepoints of candida infection will provide valuable insights for the clinical management of patients.

It has been suggested that different TLRs synergistically activate immune cells to, for instance, induce the expression of several proinflammatory molecules through the cooperation of NF-KB, IRF, STAT, MAPK, ITAM, and PI3K signaling pathways ${ }^{68-70}$. On the one hand, TLR-induced NF-KB signaling promotes the production of several key cytokines including IFNs that activate the STAT1-mediated signaling pathway ${ }^{71}$. On the other hand, IFN-y increases the expression of genes encoding TLRs $^{72-75}$. IFNs also potentiate TLR-induced gene transcription by creating a primed chromatin environment by histone acetylation that allows sustained occupancy of the transcription factors STAT1 and IRF-1 at promotors and enhancers at the $T N F, I L-6$, and $I L 12 B$ loci $^{53}$. Thus, our phenomenological study confirms these previously reported mechanistic studies and provides new insights into the molecular network of TLR and IFN signaling pathways in anti-Candida immune response. These networks also need to be investigated in other mycoses (paracoccidioidomycosis, histoplasmosis, and cryptococcosis) and other neglected diseases (Dengue, Zika, leishmaniasis, and Chagas disease) occurring in developing countries ${ }^{76}$. The TLR and IFN interactome involve more complex events than previously thought, demanding further bottom-up and top-down systems immunology investigations.

Our conclusions are based on the integration of publicly available human transcriptomes that identified common DEGs, and biological processes and signaling pathways consistently modulated across several leukocyte subpopulations in response to fungal pathogens (Candida spp.). Among these DEGs, we highlight those involved in IFN- $\alpha / \beta$ (e.g., ISGs, IRFs, SOCS, and GBPs), TLR3,4,7/8,9, and TRAF-mediated NF-KB signaling cascades. The correlation levels of DEGs involved in these signature clusters increased upon stimulation with $C$. albicans. Of note, among the consistently identified DEGs are those previously 
associated with IEl that increase the host susceptibility to fungal infections such as those causing chronic mucocutaneous candidiasis. Furthermore, these DEGs are also involved with immunological pathways related to the development of IEl phenocopies such as those targeted by anti-IL-17 or antiIL17RA autoantibodies,resulting in increased susceptibility to Candida spp. infections. Because the outcome of fungal infections depends primarily on the host immune response, it is most relevant to review those IEls that predispose to Candida infections ${ }^{14,52}$. IEls represent an essential research field that has been most useful for investigating human susceptibility models to infection, often revealing the nonredundant role of genes involved in immunologic homeostasis ${ }^{77-79}$. Of the 416 molecular defined IEI recently summarized by the expert committee of the IUIS, more than 20 syndromes were recognized to be associated with susceptibility to fungal infections ${ }^{43}$. This list of genes associated with increased risk of fungal infections includes genes regulating signaling via the IL-2 receptor, NF-kB activation, IFN induced signaling, activation of STATs, and TLR signaling. Thus, these observations support the relevance of the interactome and interplay events characterized by our analysis, increasing the understanding of consistent immunologic pathways essential for the immune response to Candida infections.

However, our manuscript has some limitations that need to be considered. For instance, the studies did not use the same $C$. albicans strains, timepoint of in vitro stimulation, multiplicity of infection (MOI), heatkilled/inactivated or alive organism, and form. Thus, these factors may differently affect the host immune response ${ }^{80,81}$, such as in the case of dataset GSE154911 ${ }^{28}$, which uses a different $C$. albicans strain (CWZ10061110) that only induced a robust transcriptional change after 24 hours of stimulation. It will be important that future studies address the impact of these factors on transcriptional dynamics in response to Candida pathogens. On the other hand, the fact that we found the activation of a network between interferon and toll-like receptor signaling across several datasets indicates that these interactions reflect an important crosstalk mechanism during the anti-candida immune response. Furthermore, only a few datasets related to Candida spp. are available compared with the large amount of data investigating other pathogens, which reinforces the need for more studies related to fungal infections.

Altogether, our work provides a systems immunology view of the interactome of anti-fungal molecules, revealing a consistent network interplay between TLR and IFN signaling pathways in response to Candida spp.. This study also indicates new biomarkers and provides novel insights into the systemic immunological mechanism against fungal infections. Future investigations dissecting this interplay will pave the way for new immunotherapy approaches to reduce the high mortalitycaused by fungal infections. Finally, our study indicates that the exploration of functional genomic approaches by applying systems immunology methods to investigate IEl will provide new opportunities to further understand the immune system in natura.

\section{Online Methods}

\section{METHODS}




\section{Datasets and curation}

We performed an integrative analysis by searching on NCBI GEO database ${ }^{82}$ and ArrayExpress database $^{83}$, to identify publicly available gene expression data of infection by $C$. albicans, $C$. auris, $C$. glabrata, C. parapsilosis, and C. tropicalis in whole blood, PBMCs, and moDCs. This search comprised studies published between March 2010 and July 2020. Since transcriptome datasets from patients with candidiasis were not publicly available we consider as criteria for inclusion: (1) gene expression data of whole blood, PBMCs, and moDCs of in vitro infection with C. albicans; (2) studies composed of at least 2 samples per group; (3) the inclusion of control groups for comparison; (4) all gene expression analysis platforms were considered; and (5) only studies that have provided the transcriptome data were included for the integrative analyses. Our exclusion criteria were (1) non-human samples, (2) treatment before molecular genetic analysis, and (3) review studies. RNAseq and MicroArray studies were included in our integrative analysis, five studies were retrieved from the NCBI GEO database ${ }^{82}$ (GSE65088 ${ }^{29}$ and GSE114180 ${ }^{30}$, GSE42606 ${ }^{11}$, GSE154911 28 , and GSE77969 ${ }^{34}$ ) and two from ArrayExpress database ${ }^{83}$ (EMTAB-13555, E-MTAB-751 ${ }^{36}$ ). Also, a single-cell RNAseq study was included ${ }^{84}$. Information about samples identification and information can be found on Suppl. Table S17 and S18.

\section{Single-cell RNASeq analysis}

We obtained the Seurat Object containing the scRNAseq data from De Vries et al. (2020) ${ }^{84}$, which was deposited in the single-cell eQTLGen Consortium database (https://eqtlgen.org/candida.html). We followed the default Seurat pipeline (https://satijalab.org/seurat/articles/pbmc3k_tutorial.html) as previously described by Stuart et al. ${ }^{85}$ to perform differential expression analysis and data visualization (UMAP, dotplot, and heatmap).

\section{Differential Expression Analysis of bulk RNAseq and microarray data}

To characterize the immunological signature from the global transcriptional profiles in infection by $C$. albicans, read counts of each RNAseq study were transformed (log2 count per million), and NetworkAnalyst 3.0 webtool (https://www.networkanalyst.ca/) ${ }^{86}$ was used to perform differential expression analysis, applying DESeq2 pipeline. The microarray studies were analyzed through GEO2R web application ${ }^{87}$, available at http://www.ncbi.nlm.nih.gov/geo/geo2r/, using limma-voom pipeline ${ }^{88}$. To select the up and downregulated genes between $C$. albicans infection and the normal group, we used the statistical cutoffs of log2 fold-change $>1$ (upregulated) or $<-1$ (downregulated) and adjusted $p$-value $<0.05$.

\section{Analysis of Gene Co-Expression Modules}

We selected the dataset GSE42606 to analyze the gene co-expression modules with the R-package CEMiTool 1.12.2 using default parameters ${ }^{24}$. 
We used the differentially expressed genes (DEGs) to identify enriched ontology terms. The pathways and the biological processes were identified through an Over-representation Analysis (ORA) and EnrichR ${ }^{89}$, and the significant enriched immunological terms were generated according to adjusted $p$-value $<0.05$. The Upset and Venn Graphs demonstrating the intersections and comparisons between common DEGs among the datasets were generated through the webtool Intervene ${ }^{90}$ and Bioinformatics \& Evolutionary Genomics (http://bioinformatics.psb.ugent.be/webtools/Venn/). We plotted the set of genes shared between the dataset in bubble-based heat maps, applying One minus cosine similarity through the webtool Morpheus (https://software.broadinstitute.org/morpheus/) ${ }^{91}$. We used ClusterProfiler ${ }^{25}$ to obtain dot plots of enriched terms associated with Candida spp.. ClusterProfiler and ORA, were performed on R software version 4.0.2 (https://www.r-project.org/index.html), through the packages DOSE, enrichplot, reactomePA, and clusterprofile ${ }^{25}$. The GOplot was plotted using the $\mathrm{R}$ packages unikn, circlize, and Goplot ${ }^{92}$. The statistical graphs were constructed using the functionalities of the ggplot2 package ${ }^{93}$. We represented the shared DEGs between different fungal infections ( $C$. albicans and $C$. auris) through circular heatmaps, using the $\mathrm{R}$ packages circlize and ComplexHeatmap ${ }^{94}$.

\section{Correlation Analysis}

We used the GSE42606 dataset to perform the correlation analysis between genes associated with TLRs as well as Type I and II IFN signaling cascades. The correlation matrices were generated with the webtool Intervene ${ }^{90}$ (https://intervene.readthedocs.io/en/latest/index.html), using Pearson coefficient. The Canonical Correlation Analysis (CCA $)^{95}$ was applied to investigate patterns of association between IFN and TLR genes from the same dataset. The CCA was performed on $\mathrm{R}$ software version 4.0.2 (https://www.r-project.org/index.html), through the packages CCA, and whitening ${ }^{95}$. Principal Component Analysis (PCA) analysis was built using R functions prcomp and princomp, through factoextra package.

\section{Molecular Network}

Networks of related pathways to fungal infection immune responses and physical protein-protein interaction (PPI) networks of DEGs found across all datasets were annotated, analyzed and visualized using NAViGaTOR 3.0,14 ${ }^{96}$. Node color represents Gene Ontology cellular component as per legend. DEGs were used as input into Integrated Interactions Database (IID version 2020-05;

http://ophid.utoronto.ca/iid) $)^{97,98}$ to identify direct physical protein interactions. Networks were exported in SVG file format, and finalized in Adobe Illustrator 2021.

\section{Multi-study Factor Analysis (MSFA)}

MSFA is a generalized version of factor analysis that allows for the joint analysis of multiple studies. MSFA estimates shared factors common to all studies, as well as factors specific to individual studies. Estimation of parameters for the MSFA model can be computed using either a frequentist or a Bayesian approach. Compared with the frequentist analysis, the Bayesian offers two major advantages: 1- it provides better-defined factors, and 2- it chooses the dimension of the common and study-specific 
factors through a practical and useful approach. We adopt the Bayesian multi-study ${ }^{99}$, for the inferential analysis to identify -common and study-specific factors ${ }^{14,33,100}$ shared by GSE65088 and GSE42606. The Bayesian MSFA considers all data at once in an integrated approach, estimating parameters by maximum-likelihood analysis ${ }^{101}$.

\section{Interferome Analysis}

The identification of interferome genes was performed with Interferome V2.01 (http://www.interferome.org/interferome/home.jspx).

\section{Single-cell RNA-seq differential expression analysis}

Seurat package was used to obtain the DEGs between the different cell types under the conditions of infection by $C$. albicans and resting cells. Enrichment of DEGs by cell group and by total DEGs was done according to the described for ClusterProfiler package.

\section{Figures layout edition}

The layout edition of figures, such as proportional Venn graphs, was made using CorelDraw 2019 (https://www.coreldraw.com/br/pages/coreldraw-2019/).

\section{Code Availability}

$\mathrm{R}$ codes used in this work are available athttps://github.com/ranieri131/SalgadoRC_CANDIDA_IMMUNE_RESPONSE_2021

\section{Data availability}

The published transcriptome datasets can be found in the GEO and Array Express databases (IDs. GSE65088, GSE114180, GSE42606, GSE154911, GSE77969, E-MTAB-135, E-MTAB-751). Single-cell data is available as Seurat Object on doi.org/10.1371/journal.ppat.1008408.

\section{References}

1. Chow, N. A. et al. Multiple introductions and subsequent transmission of multidrug-resistant Candida auris in the USA: a molecular epidemiological survey. Lancet Infect. Dis. 18, 1377-1384 (2018).

2. World Health Organization. GLASS report: early implementation 2017-2018. https://www.who.int/publications/i/item/9789241515061 (2019).

3. Casadevall, A. Don't Forget the Fungi When Considering Global Catastrophic Biorisks. Heal. Secur. 15, 341-342 (2017). 
4. Warnock, D. W. Fungal diseases: an evolving public health challenge. Med. Mycol. 44, 697-705 (2006).

5. Meis, J. F. \& Chowdhary, A. Candida auris: a global fungal public health threat. Lancet Infect. Dis. 18, 1298-1299 (2018).

6. Zeng, Z. et al. Surveillance study of the prevalence, species distribution, antifungal susceptibility, risk factors and mortality of invasive candidiasis in a tertiary teaching hospital in Southwest China. BMC Infect. Dis. 19, 939 (2019).

7. Kullberg, B. J. \& Arendrup, M. C. Invasive Candidiasis. N. Engl. J. Med. 373, 1445-1456 (2015).

8. Lee, P. P. \& Lau, Y.-L. Cellular and Molecular Defects Underlying Invasive Fungal InfectionsRevelations from Endemic Mycoses. Front. Immunol. 8, (2017).

9. Cheng, S.-C., Joosten, L. A. B., Kullberg, B.-J. \& Netea, M. G. Interplay between Candida albicans and the Mammalian Innate Host Defense. Infect. Immun. 80, 1304-1313 (2012).

10. Johnson, M. D. et al. Cytokine Gene Polymorphisms and the Outcome of Invasive Candidiasis: A Prospective Cohort Study. Clin. Infect. Dis. 54, 502-510 (2012).

11. Smeekens, S. P. et al. Functional genomics identifies type I interferon pathway as central for host defense against Candida albicans. Nat. Commun. 4, 1342 (2013).

12. Romani, L. Immunity to fungal infections. Nat. Rev. Immunol. 11, 275-288 (2011).

13. Patin, E. C., Thompson, A. \& Orr, S. J. Pattern recognition receptors in fungal immunity. Semin. Cell Dev. Biol. 89, 24-33 (2019).

14. Netea, M. G., Joosten, L. A. B., van der Meer, J. W. M., Kullberg, B.-J. \& van de Veerdonk, F. L. Immune defence against Candida fungal infections. Nat. Rev. Immunol. 15, 630-642 (2015).

15. Liu, T., Zhang, L., Joo, D. \& Sun, S.-C. NF-kB signaling in inflammation. Signal Transduct. Target. Ther. 2, 17023 (2017).

16. Warnatsch, A. et al. Reactive Oxygen Species Localization Programs Inflammation to Clear Microbes of Different Size. Immunity 46, 421-432 (2017).

17. Kolaczkowska, E. \& Kubes, P. Neutrophil recruitment and function in health and inflammation. Nat. Rev. Immunol. 13, 159-175 (2013).

18. Urban, C. F., Reichard, U., Brinkmann, V. \& Zychlinsky, A. Neutrophil extracellular traps capture and kill Candida albicans yeast and hyphal forms. Cell. Microbiol. 8, 668-676 (2006).

19. Romani, L. Immunity to fungal infections. Nat. Rev. Immunol. 4, 11-24 (2004). 
20. Schimke, L. F. et al. Paracoccidioidomycosis Associated with a Heterozygous STAT4 Mutation and Impaired IFN-y Immunity. in Journal of Infectious Diseases vol. 216 1623-1634 (Oxford University Press, 2017).

21. Seurat - Guided Clustering Tutorial. https://satijalab.org/seurat/articles/pbmc3k_tutorial.html (2021).

22. Chaussabel, D. \& Baldwin, N. Democratizing systems immunology with modular transcriptional repertoire analyses. Nat. Rev. Immunol. 14, 271-280 (2014).

23. WGCNA package: Frequently Asked Questions.

https://horvath.genetics.ucla.edu/html/CoexpressionNetwork/Rpackages/WGCNA/faq.html.

24. Russo, P. S. T. et al. CEMiTool: a Bioconductor package for performing comprehensive modular coexpression analyses. BMC Bioinformatics 19, 56 (2018).

25. Yu, G., Wang, L. G., Han, Y. \& He, Q. Y. ClusterProfiler: An R package for comparing biological themes among gene clusters. Omi. A J. Integr. Biol. 16, 284-287 (2012).

26. Bruno, M. et al. Data of common and species-specific transcriptional host responses to pathogenic fungi. Data Br. 35, 106928 (2021).

27. Jaeger, M. et al. The RIG-I-like helicase receptor MDA5 (IFIH1) is involved in the host defense against Candida infections. Eur. J. Clin. Microbiol. Infect. Dis. 34, 963-974 (2015).

28. Bruno, M. et al. Transcriptional and functional insights into the host immune response against the emerging fungal pathogen Candida auris. Nat. Microbiol. 5, 1516-1531 (2020).

29. Dix, A. et al. Biomarker-based classification of bacterial and fungal whole-blood infections in a genome-wide expression study. Front. Microbiol. 6, 171 (2015).

30. Sieber, P. et al. Comparative Study on Alternative Splicing in Human Fungal Pathogens Suggests Its Involvement During Host Invasion. Front. Microbiol. 9, 2313 (2018).

31. Kämmer, P. et al. Survival Strategies of Pathogenic Candida Species in Human Blood Show Independent and Specific Adaptations. MBio 11, (2020).

32. Ramesh, N., Salama, M., Dangott, B. \& Tasdizen, T. Isolation and two-step classification of normal white blood cells in peripheral blood smears. J. Pathol. Inform. 3, 13 (2012).

33. De Vito, R., Bellio, R., Trippa, L. \& Parmigiani, G. Multi-study factor analysis. Biometrics 75, 337346 (2019).

34. Dix, A. et al. Specific and Novel microRNAs Are Regulated as Response to Fungal Infection in Human Dendritic Cells. Front. Microbiol. 8, 270 (2017). 
35. Rizzetto, L. et al. Differential IL-17 production and mannan recognition contribute to fungal pathogenicity and commensalism. J. Immunol. 184, 4258-68 (2010).

36. Rizzetto, L. et al. The modular nature of dendritic cell responses to commensal and pathogenic fungi. PLoS One 7, e42430 (2012).

37. Bourgeois, C. et al. Conventional Dendritic Cells Mount a Type I IFN Response against Candida spp. Requiring Novel Phagosomal TLR7-Mediated IFN- $\beta$ Signaling. J. Immunol. 186, 3104-3112 (2011).

38. Romani, L. \& Puccetti, P. Controlling pathogenic inflammation to fungi. Expert Rev. Anti. Infect. Ther. 5, 1007-1017 (2007).

39. Romani, L., Zelante, T., De Luca, A., Fallarino, F. \& Puccetti, P. IL-17 and Therapeutic Kynurenines in Pathogenic Inflammation to Fungi. J. Immunol. 180, 5157-5162 (2008).

40. Aggarwal, R. \& Ranganathan, P. Common pitfalls in statistical analysis: The use of correlation techniques. Perspect. Clin. Res. 7, 187 (2016).

41. Rickman, J. M., Wang, Y., Rollett, A. D., Harmer, M. P. \& Compson, C. Data analytics using canonical correlation analysis and Monte Carlo simulation. npj Comput. Mater. 3, 26 (2017).

42. Arendrup, M. C. \& Patterson, T. F. Multidrug-Resistant Candida: Epidemiology, Molecular Mechanisms, and Treatment. J. Infect. Dis. 216, S445-S451 (2017).

43. Tangye, S. G. et al. Human Inborn Errors of Immunity: 2019 Update on the Classification from the International Union of Immunological Societies Expert Committee. J. Clin. Immunol. 40, 24-64 (2020).

44. Vallabhajosyula, R. R., Chakravarti, D., Lutfeali, S., Ray, A. \& Raval, A. Identifying Hubs in Protein Interaction Networks. PLoS One 4, e5344 (2009).

45. Kawai, T. \& Akira, S. Signaling to NF-kB by Toll-like receptors. Trends Mol. Med. 13, 460-469 (2007).

46. Tsai, M.-H., Pai, L.-M. \& Lee, C.-K. Fine-Tuning of Type I Interferon Response by STAT3. Front. Immunol. 10, (2019).

47. Balic, J. J. et al. STAT3 serine phosphorylation is required for TLR4 metabolic reprogramming and IL-1 $\beta$ expression. Nat. Commun. 11, 3816 (2020).

48. Luu, K. et al. STAT1 plays a role in TLR signal transduction and inflammatory responses. Immunol. Cell Biol. 92, 761-769 (2014).

49. Ramana, C. V, Gil, M. P., Schreiber, R. D. \& Stark, G. R. Stat1-dependent and-independent pathways in IFN- $\gamma$-dependent signaling. Trends Immunol. 23, 96-101 (2002). 
50. Rhee, S. H., Jones, B. W., Toshchakov, V., Vogel, S. N. \& Fenton, M. J. Toll-like Receptors 2 and 4 Activate STAT1 Serine Phosphorylation by Distinct Mechanisms in Macrophages. J. Biol. Chem. 278, 22506-22512 (2003).

51. Hardison, S. E. \& Brown, G. D. C-type lectin receptors orchestrate antifungal immunity. Nat. Immunol. 13, 817-822 (2012).

52. Muñoz, J. F. et al. Coordinated host-pathogen transcriptional dynamics revealed using sorted subpopulations and single macrophages infected with Candida albicans. Nat. Commun. 10, 1607 (2019).

53. Qiao, Y. et al. Synergistic Activation of Inflammatory Cytokine Genes by Interferon-y-Induced Chromatin Remodeling and Toll-like Receptor Signaling. Immunity 39, 454-469 (2013).

54. Donini, M., Zenaro, E., Tamassia, N. \& Dusi, S. NADPH oxidase of human dendritic cells: Role inCandida albicans killing and regulation by interferons, dectin-1 and CD206. Eur. J. Immunol. 37, 11941203 (2007).

55. Riedelberger, M. et al. Type I Interferons Ameliorate Zinc Intoxication of Candida glabrata by Macrophages and Promote Fungal Immune Evasion. iScience 23, 101121 (2020).

56. Riedelberger, M. et al. Type I Interferon Response Dysregulates Host Iron Homeostasis and Enhances Candida glabrata Infection. Cell Host Microbe 27, 454-466.e8 (2020).

57. Patin, E. C. et al. IL-27 Induced by Select Candida spp. via TLR7/NOD2 Signaling and IFN$\beta$ Production Inhibits Fungal Clearance. J. Immunol. 197, 208-221 (2016).

58. Cardone, M. et al. Interleukin-1 and Interferon- $\gamma$ Orchestrate $\beta$-Glucan-Activated Human Dendritic Cell Programming via IKB-ל Modulation. PLoS One 9, e114516 (2014).

59. del Fresno, C. et al. Interferon- $\beta$ Production via Dectin-1-Syk-IRF5 Signaling in Dendritic Cells Is Crucial for Immunity to C. albicans. Immunity 38, 1176-1186 (2013).

60. Biondo, C. et al. Recognition of yeast nucleic acids triggers a host-protective type I interferon response. Eur. J. Immunol. 41, 1969-1979 (2011).

61. Li, T., Niu, X., Zhang, X., Wang, S. \& Liu, Z. Recombinant Human IFNa-2b Response Promotes Vaginal Epithelial Cells Defense against Candida albicans. Front. Microbiol. 8, (2017).

62. Li, T., Liu, Z., Zhang, X., Chen, X. \& Wang, S. Therapeutic effectiveness of type I interferon in vulvovaginal candidiasis. Microb. Pathog. 134, 103562 (2019).

63. Pekmezovic, M. et al. Candida pathogens induce protective mitochondria-associated type I interferon signalling and a damage-driven response in vaginal epithelial cells. Nat. Microbiol. 6, 643-657 (2021). 
64. Majer, O. et al. Type I Interferons Promote Fatal Immunopathology by Regulating Inflammatory Monocytes and Neutrophils during Candida Infections. PLoS Pathog. 8, e1002811 (2012).

65. Break, T. J. et al. Aberrant type 1 immunity drives susceptibility to mucosal fungal infections. Science (80- ). 371, eaay5731 (2021).

66. Okada, S. et al. Human STAT1 Gain-of-Function Heterozygous Mutations: Chronic Mucocutaneous Candidiasis and Type I Interferonopathy. J. Clin. Immunol. 40, 1065-1081 (2020).

67. Stawowczyk, M. et al. Pathogenic Effects of IFIT2 and Interferon- $\beta$ during Fatal Systemic Candida albicans Infection. MBio 9, (2018).

68. Hu, X., Chen, J., Wang, L. \& Ivashkiv, L. B. Crosstalk among Jak-STAT, Toll-like receptor, and ITAMdependent pathways in macrophage activation. J. Leukoc. Biol. 82, 237-243 (2007).

69. Bohnenkamp, H. R., Papazisis, K. T., Burchell, J. M. \& Taylor-Papadimitriou, J. Synergism of Tolllike receptor-induced interleukin-12p70 secretion by monocyte-derived dendritic cells is mediated through p38 MAPK and lowers the threshold of T-helper cell type I responses. Cell. Immunol. 247, 72-84 (2007).

70. Makela, S. M., Strengell, M., Pietila, T. E., Osterlund, P. \& Julkunen, I. Multiple signaling pathways contribute to synergistic TLR ligand-dependent cytokine gene expression in human monocyte-derived macrophages and dendritic cells. J. Leukoc. Biol. 85, 664-672 (2009).

71. Tong, Y. et al. Enhanced TLR-induced NF-KB signaling and type I interferon responses in NLRC5 deficient mice. Cell Res. 22, 822-835 (2012).

72. Bosisio, D. et al. Stimulation of toll-like receptor 4 expression in human mononuclear phagocytes by interferon-ү: a molecular basis for priming and synergism with bacterial lipopolysaccharide. Blood $\mathbf{9 9}$, 3427-3431 (2002).

73. Schroder, K. et al. PU.1 and ICSBP control constitutive and IFN-Y-regulated TIr9 gene expression in mouse macrophages. J. Leukoc. Biol. 81, 1577-1590 (2007).

74. Kajita, A. i. et al. Interferon-Gamma Enhances TLR3 Expression and Anti-Viral Activity in Keratinocytes. J. Invest. Dermatol. 135, 2005-2011 (2015).

75. Mita, Y. et al. Toll-like receptor 4 surface expression on human monocytes and B cells is modulated by IL-2 and IL-4. Immunol. Lett. 81, 71-75 (2002).

76. Rodrigues, M. L. \& Nosanchuk, J. D. Fungal diseases as neglected pathogens: A wake-up call to public health officials. PLoS Negl. Trop. Dis. 14, e0007964 (2020).

77. Casanova, J.-L. \& Abel, L. Lethal Infectious Diseases as Inborn Errors of Immunity: Toward a Synthesis of the Germ and Genetic Theories. Annu. Rev. Pathol. Mech. Dis. 16, 23-50 (2021). 
78. Notarangelo, L. D., Bacchetta, R., Casanova, J.-L. \& Su, H. C. Human inborn errors of immunity: An expanding universe. Sci. Immunol. 5, eabb1662 (2020).

79. Casanova, J.-L. \& Abel, L. The human model: a genetic dissection of immunity to infection in natural conditions. Nat. Rev. Immunol. 4, 55-66 (2004).

80. Joly, S. et al. Cutting Edge: Candida albicans Hyphae Formation Triggers Activation of the Nlrp3 Inflammasome. J. Immunol. 183, 3578-3581 (2009).

81. Schönherr, F. A. et al. The intraspecies diversity of C. albicans triggers qualitatively and temporally distinct host responses that determine the balance between commensalism and pathogenicity. Mucosal Immunol. 10, 1335-1350 (2017).

82. Edgar, R., Domrachev, M. \& Lash, A. E. Gene Expression Omnibus: NCBI gene expression and hybridization array data repository. Nucleic Acids Res. 30, 207-210 (2002).

83. Athar, A. et al. ArrayExpress update - from bulk to single-cell expression data. Nucleic Acids Res. 47, D711-D715 (2019).

84. de Vries, D. H. et al. Integrating GWAS with bulk and single-cell RNA-sequencing reveals a role for LY86 in the anti-Candida host response. PLOS Pathog. 16, e1008408 (2020).

85. Stuart, T. et al. Comprehensive Integration of Single-Cell Data. Cell 177, 1888-1902.e21 (2019).

86. Zhou, G. et al. NetworkAnalyst 3.0: a visual analytics platform for comprehensive gene expression profiling and meta-analysis. Nucleic Acids Res. 47, W234-W241 (2019).

87. Barrett, T. et al. NCBI GEO: archive for functional genomics data sets-update. Nucleic Acids Res. 41, D991-D995 (2012).

88. Law, C. W., Chen, Y., Shi, W. \& Smyth, G. K. voom: precision weights unlock linear model analysis tools for RNA-seq read counts. Genome Biol. 15, R29 (2014).

89. Kuleshov, M. V. et al. Enrichr: a comprehensive gene set enrichment analysis web server 2016 update. Nucleic Acids Res. 44, W90-W97 (2016).

90. Khan, A. \& Mathelier, A. Intervene: a tool for intersection and visualization of multiple gene or genomic region sets. BMC Bioinformatics 18, 287 (2017).

91. Starruß, J., de Back, W., Brusch, L. \& Deutsch, A. Morpheus: a user-friendly modeling environment for multiscale and multicellular systems biology. Bioinformatics 30, 1331-1332 (2014).

92. Walter, W., Sánchez-Cabo, F. \& Ricote, M. GOplot: an R package for visually combining expression data with functional analysis: Fig. 1. Bioinformatics 31, 2912-2914 (2015). 
93. Wickham, H. Getting Started with ggplot2. in 11-31 (2016). doi:10.1007/978-3-319-24277-4_2.

94. Gu, Z., Eils, R. \& Schlesner, M. Complex heatmaps reveal patterns and correlations in multidimensional genomic data. Bioinformatics 32, 2847-2849 (2016).

95. Jendoubi, T. \& Strimmer, K. A whitening approach to probabilistic canonical correlation analysis for omics data integration. BMC Bioinformatics 20, 15 (2019).

96. Brown, K. R. et al. NAViGaTOR: Network Analysis, Visualization and Graphing Toronto. Bioinformatics 25, 3327-3329 (2009).

97. Kotlyar, M., Pastrello, C., Malik, Z. \& Jurisica, I. IID 2018 update: context-specific physical proteinprotein interactions in human, model organisms and domesticated species. Nucleic Acids Res. 47, D581D589 (2019).

98. Pastrello, C., Kotlyar, M. \& Jurisica, I. Informed Use of Protein-Protein Interaction Data: A Focus on the Integrated Interactions Database (IID). in 125-134 (2020). doi:10.1007/978-1-4939-9873-9_10.

99. De Vito, R., Bellio, R., Trippa, L. \& Parmigiani, G. Bayesian Multi-study Factor Analysis for Highthroughput Biological Data. (2018).

100. Cabral-Marques, O. et al. GPCR-specific autoantibody signatures are associated with physiological and pathological immune homeostasis. Nat. Commun. 9, 5224 (2018).

101. Dempster, A. P., Laird, N. M. \& Rubin, D. B. Maximum Likelihood from Incomplete Data Via the EM Algorithm . J. R. Stat. Soc. Ser. B 39, 1-22 (1977).

\section{Declarations}

\section{Acknowledgments}

We acknowledge the São Paulo Research Foundation (FAPESP grants 2018/18886-9, 2020/01688-0, and 2020/07069-0 to OCM) for financial support. RCS is financed by the national council for scientific and technological development (CNPQ). Computational analysis was supported by FAPESP and partially by the grants from Ontario Research Fund (\#34876), Natural Sciences Research Council (NSERC \#203475), Canada Foundation for Innovation (CFI \#29272, \#225404, \#33536), and IBM. This study was financed in part by the coordination for the improvement of higher education personnel - Brazil (CAPES) - finance code 001.

We would like to acknowledge all the investigators who made their datasets publicly available by depositing them in GEO and ArrayExpress.

\section{Author Contributions}


RCS, PPF, OCM co-wrote the manuscript; RCS, DLMF, TTF, PPF, GCM, NOSC, VLGC, LFS, OCM provided scientific insights; RCS, DLMF, AHCM, SMSN, KTA, CASP, GCB, DRP, ISF, RV, IJ, and OCM performed bioinformatics analyses; RCS, DLMF, and OCM conceived and designed the study; RCS, TTF, LFS, PPF, NOSC, VLGC, HDO, LFS, IJ, ACN, and OCM revised and edited the final manuscript; ACN and OCM supervised the project.

\section{Competing interest statement}

The authors declare no competing financial and/or non-financial interests concerning the work described.

\section{Figures}


Fig. 1

a

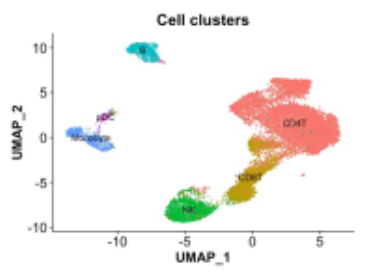

b

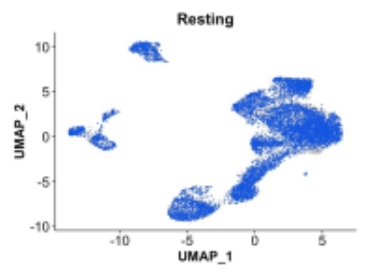

c

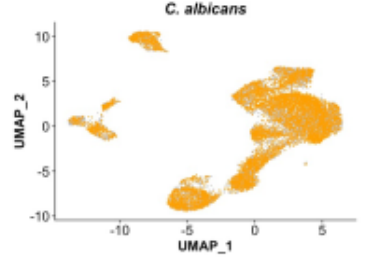

d

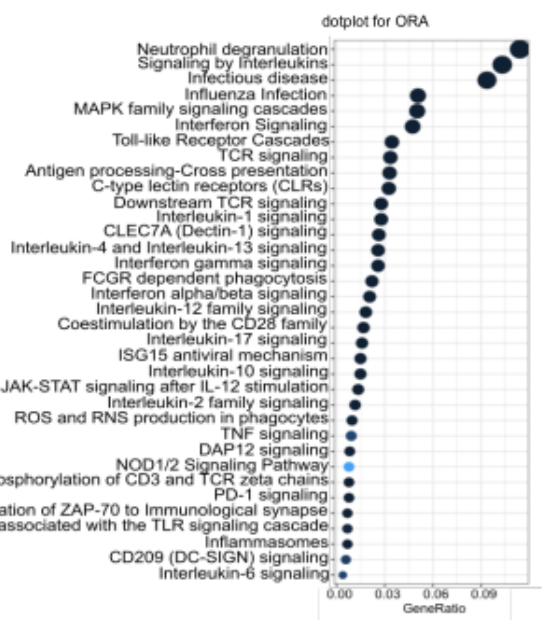

e

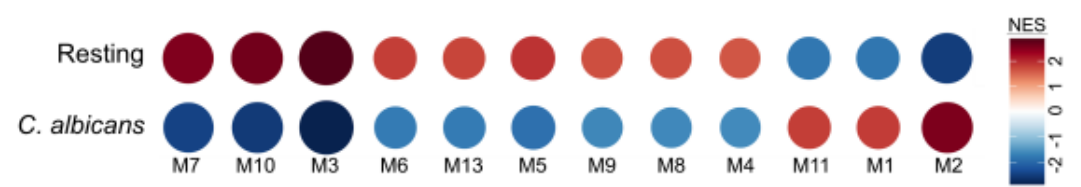

f

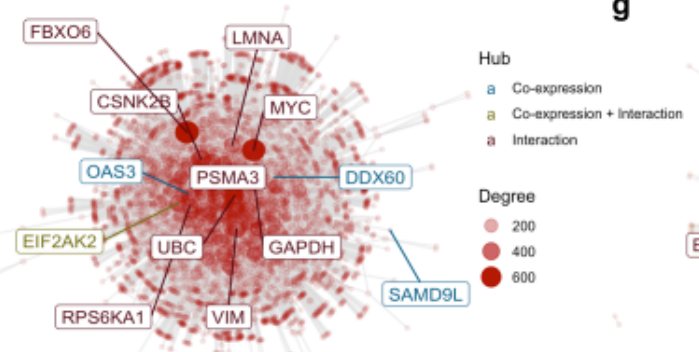

h

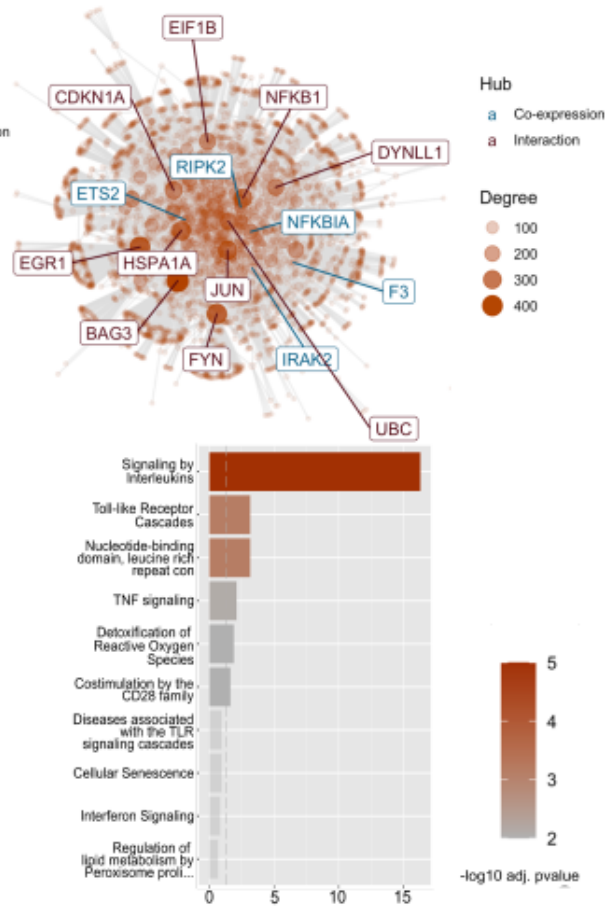

\section{Figure 1}

Multi-layered induction of TLR and IFN signaling pathways in response to C. albicans. a, UMAP visualization of scRNAseq profiles colored according to cell clusters. b, c, UMAP of resting and C. albicans-activated cells groups. DEGs, Differentially Expressed Genes; IFN, Interferon; ORA, Over representation analysis; scRNAseq, single-cell RNA sequencing; TLR, Toll-like Receptor; UMAP, Uniform Manifold Approximation and Projection. d, Dot plot showing pathways associated with immune response 
to C. albicans, obtained by ORA of DEGs. e, Co-expression modules significantly enriched (M1-M11, and M13) in PBMCs (resting $n=30 ; C$. albicans infected $n=24$; dataset GSE42606). $f$ and $g$ Network representation of M1 and M2 with hubs (most connected genes) colored based on co-expression (blue color), co-expressed and interactions (green color), or only interactions (dark-red color). $\mathrm{h}$ and $\mathrm{i}$, Enrichment representation obtained by modular genes co-expression in $\mathrm{M} 1$ and $\mathrm{M} 2$ showing significantly (-Log10 transformed adjusted p-value) enriched signaling pathways. IFN, Interferon; TLR, Toll-like Receptor.

Fig. 2
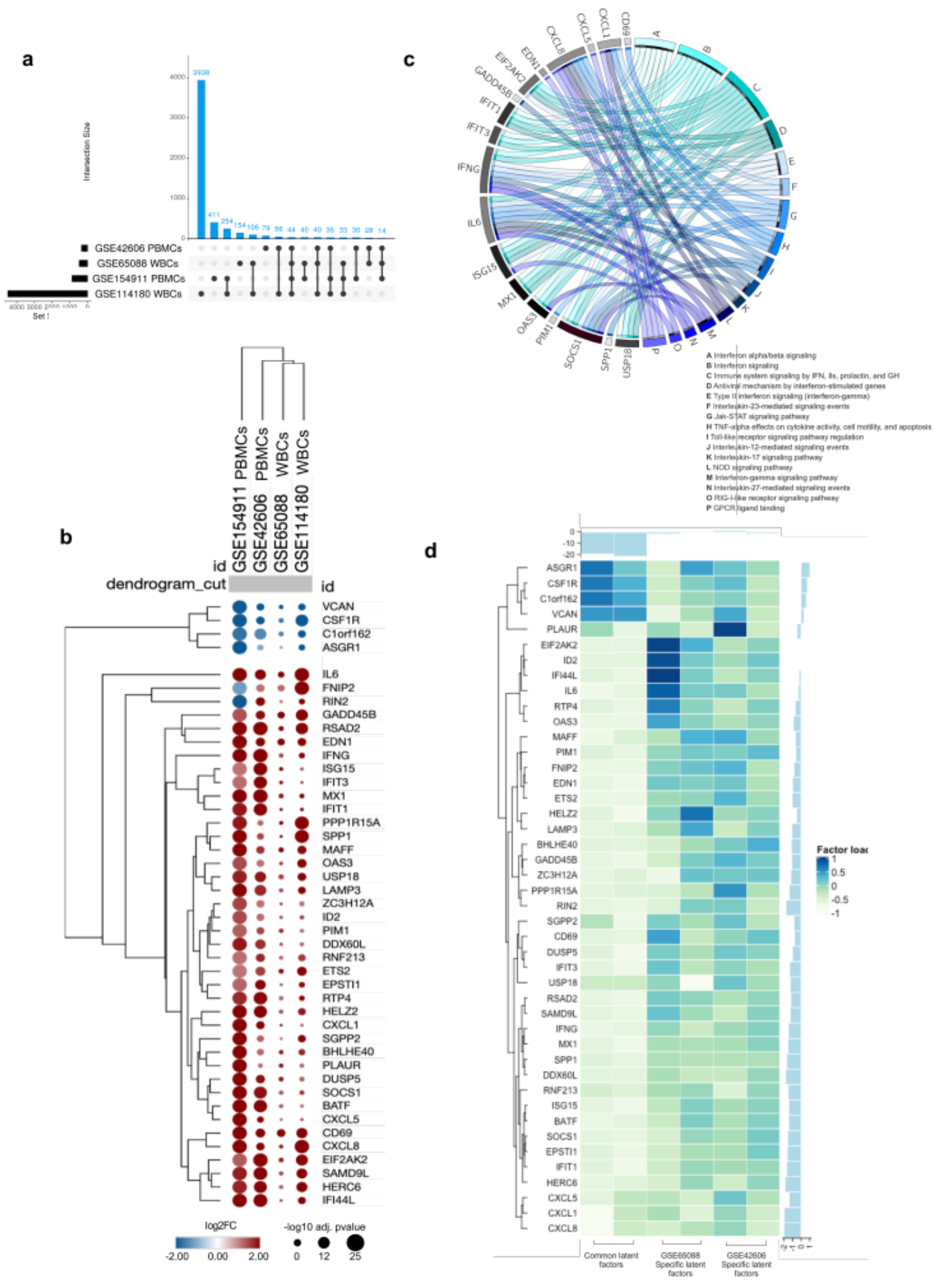


\section{Figure 2}

C. albicans activates common TLR- and IFN-associated genes in peripheral blood leukocytes. a, The upset plot displays the number (set size) of DEGs present in each dataset ( $y$-axis: WBCs, GSE65088, and GSE114180; PBMCs: GSE42606 and GSE154911) and their intersections. Black bubbles, present in the rows, mark the dataset which refers to the amount present in the blue columns, with intersections between two or more groups being shown. b, Hierarchical clustering of the 44 common DEGs demonstrating gene expression patterns across the different studies. The size and color of circles correspond to -Log10 transformed adjusted p-value and Log2 fold change (Log2FC), respectively. Blue represents downregulated and red indicates up-regulated DEGs. The cut-off applied to identify the down-/upregulated genes was Log2FC $<-1 />1$ and adjusted p-value $<0.05$. Rows and columns were clustered based on cosine similarity between Log2FC values. c, GOplot of selected immunological pathways and associated gene. $d$, Heatmap of common and specific latent factors across the studies. Heatmaps contain genes presenting positive and negative loadings ranging from -1 to 1. DEGs, Differentially Expressed Genes; PBMCs, Peripheral Blood Mononuclear Cells; WBCs, White Blood Cells. 
Fig. 3

a

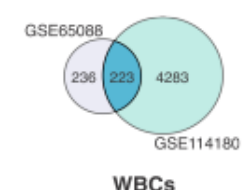

b

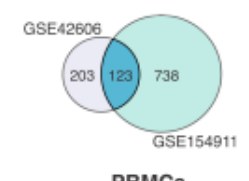

PBMCs
C

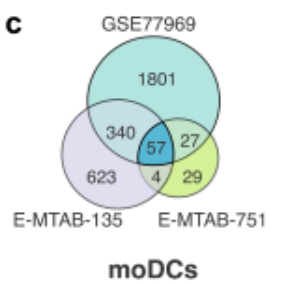

d

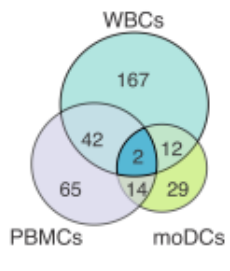

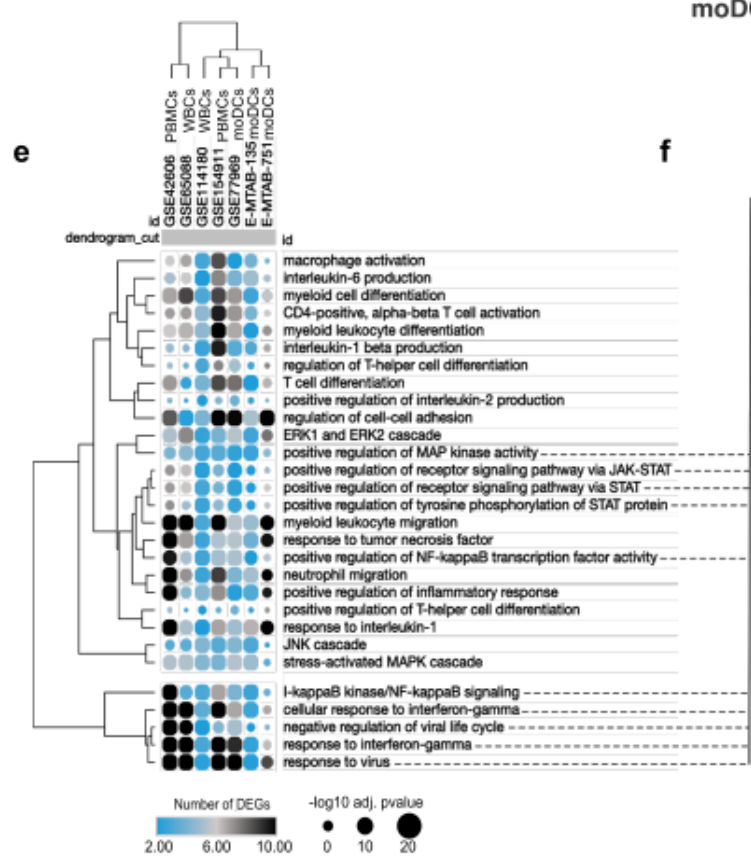

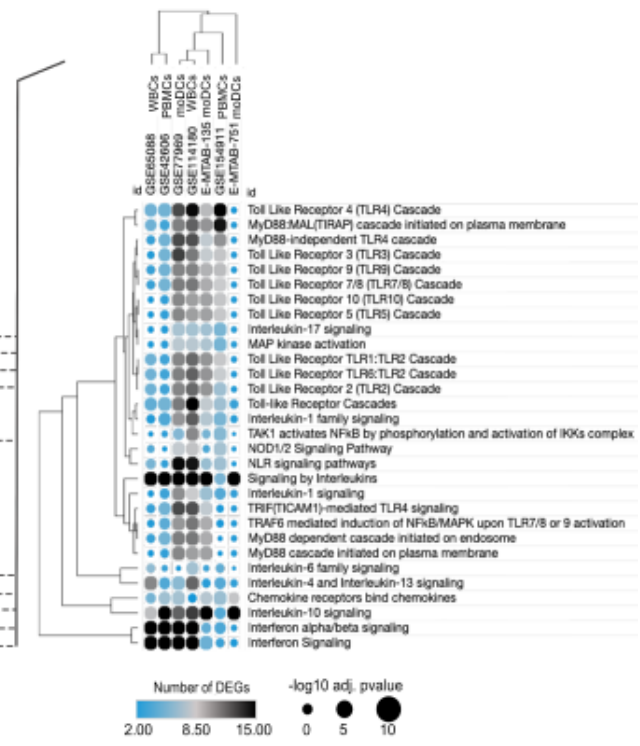

g

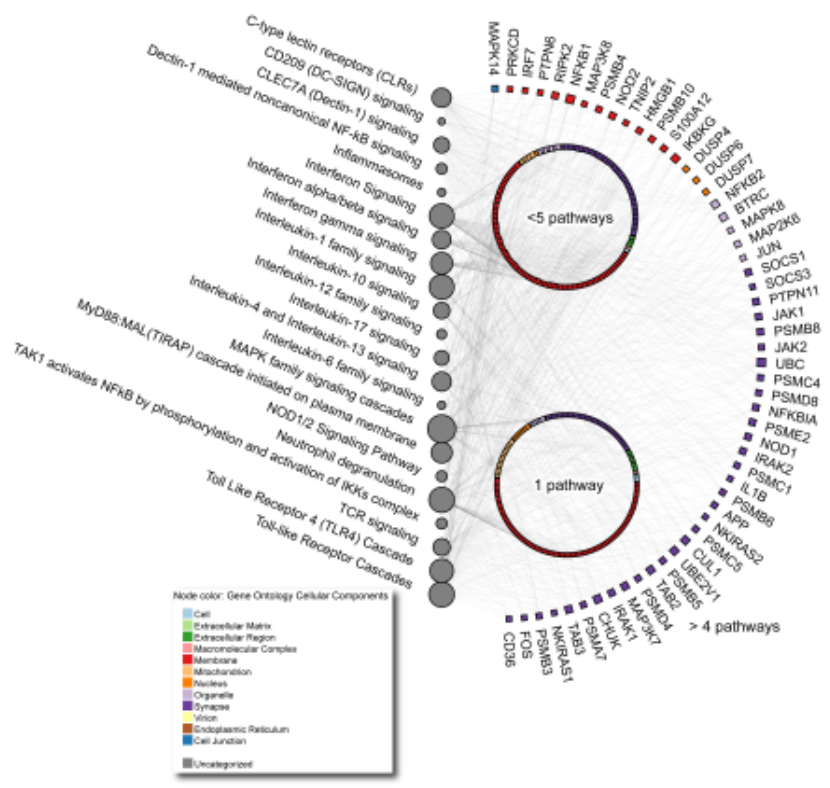

\section{Figure 3}

C. albicans activate common TLR and IFN signaling pathways across different leukocyte populations. ac, Proportional Venn diagrams displaying the number of DEGs present in each dataset grouped by cell type and their intersections: datasets of WBCs (a), PBMCs (b), and moDCs (c). d, The intersection plot highlights the number of common DEGs across different cell groups (Venn diagrams were created using CorelDraw2019, available at coreldraw.com). e, Hierarchical clustering exhibiting the pathways enriching 
common biological processes across the studies (Suppl. Table S10). f, Further analysis of TLR- and IFNassociated pathways. In both heatmaps the size of circles corresponds to adjusted p-value transformed into -Log10 and color intensity indicates the number of genes in each biological process and pathway across the studies, respectively. $g$, Network demonstrating the interactions between TLR- and IFNassociated DEGs/signaling pathways with other molecules and signaling cascades classically associated with the antifungal immune responses. Enrichment analysis was performed using Reactome. Circular nodes represent pathways and their size denote the number of genes enriching the pathways. Colored squares represent the cellular location of genes. Genes interacting with more than 5 pathways are named. The interaction network was build using the NAViGaTOR software. DEGs, Differentially Expressed Genes; moDCs, Monocyte-Derived Dendritic Cells; IFN, Interferon; PBMCs, Peripheral Blood Mononuclear Cells; TLR, Toll-like Receptor; WBCs, White Blood Cells. 
a
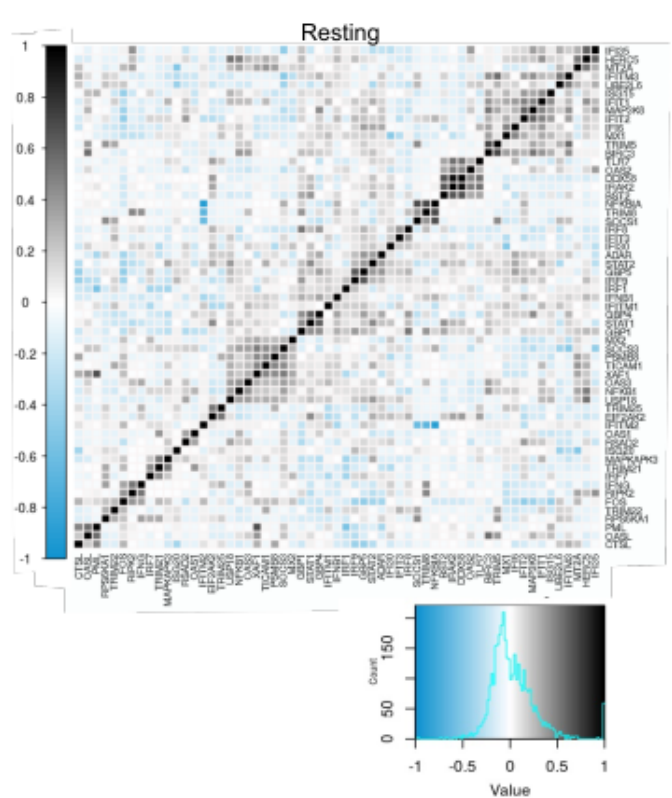

b
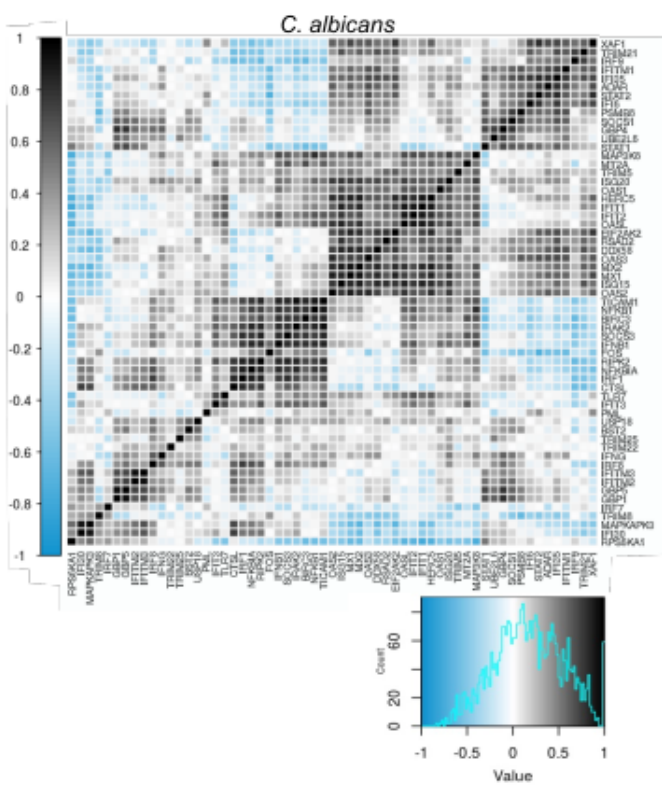

C

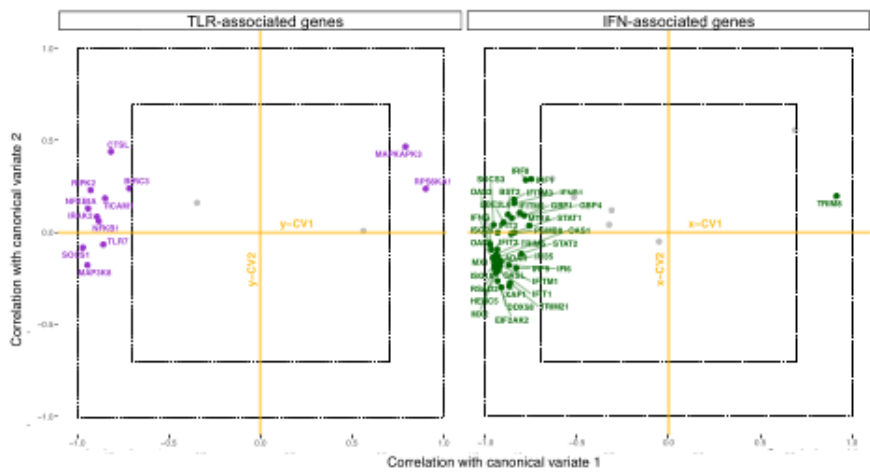

d
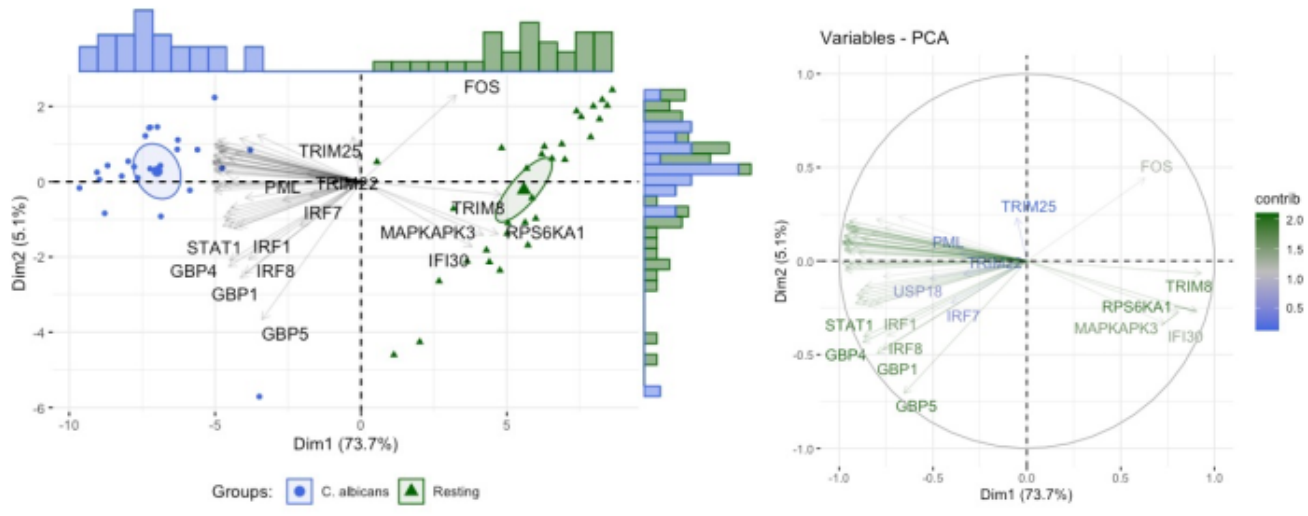

Figure 4

Relationship between molecules associated with TLR and IFN signaling cascades. a,b, Correloplot of DEGs associated with TLR and IFN signaling cascades in PBMCs (GSE42606) in the $a$, absence or $b$, presence of $\mathrm{C}$. albicans. Histograms of Pearson's correlation coefficient, containing negative and positive correlation from 1 to -1 , respectively. c, Estimated correlations of TLR - and IFN -associated DEGs versus their corresponding first 2 canonical variates (x-CV1 and x-CV2, for IFN- associated genes; $y$-CV1 and $y$ - 
CV2 for TLR-associated genes). Grey colored variables (with names omitted) are those with correlation coefficient $\leq 0.7$ in its two corresponding canonical variates. Inner dotted lines limit the canonical correlation coefficient between -0.7 and 0.7 , while outer dotted lines between -1 and 1 . d, e, PCA was used for stratification analysis of resting and $\mathrm{C}$. albicans infected PBMCs based on TLR- and IFN-associated DEGs. $d$, Of note, individuals with similar expression values for these DEGs are grouped together; $\mathrm{e}$, Variables with positive correlation are pointing to same side of the plot, contrasting with negative correlated variables, which point to opposite sides. DEGs, Differentially Expressed Genes; IFN, Interferon; PBMCs, Peripheral Blood Mononuclear Cells; TLR, Toll-like Receptor. 

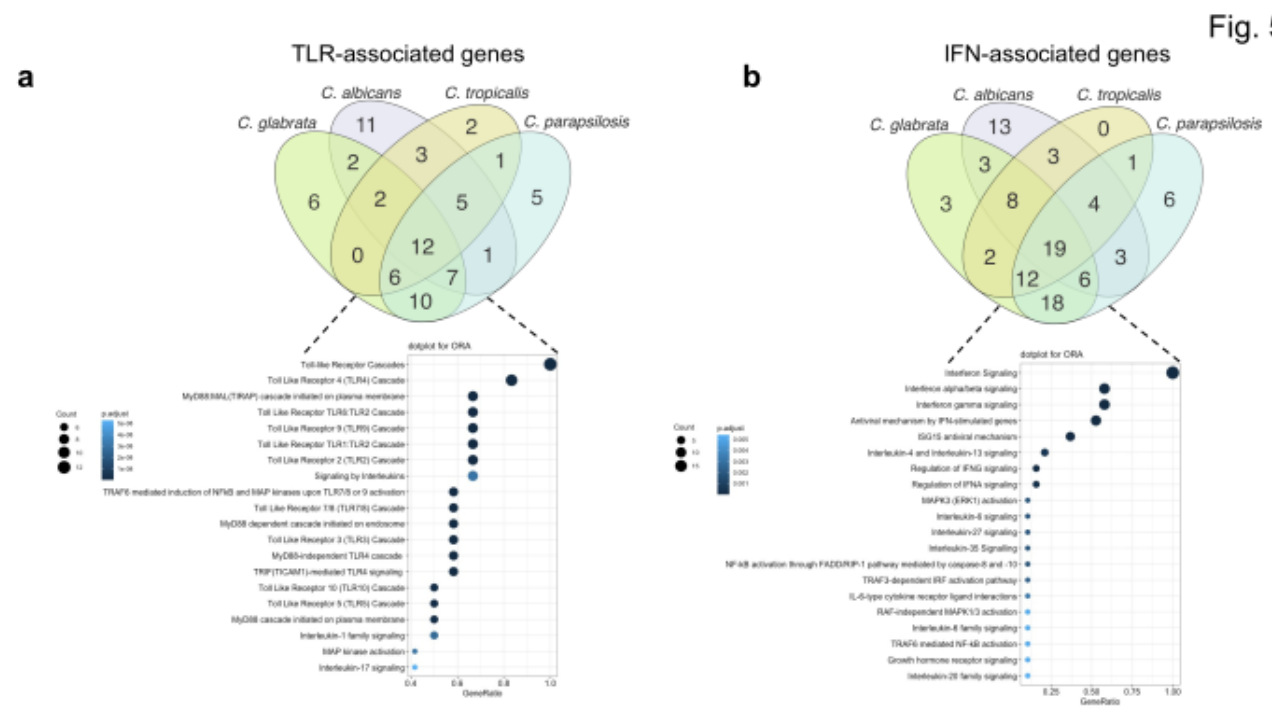

c

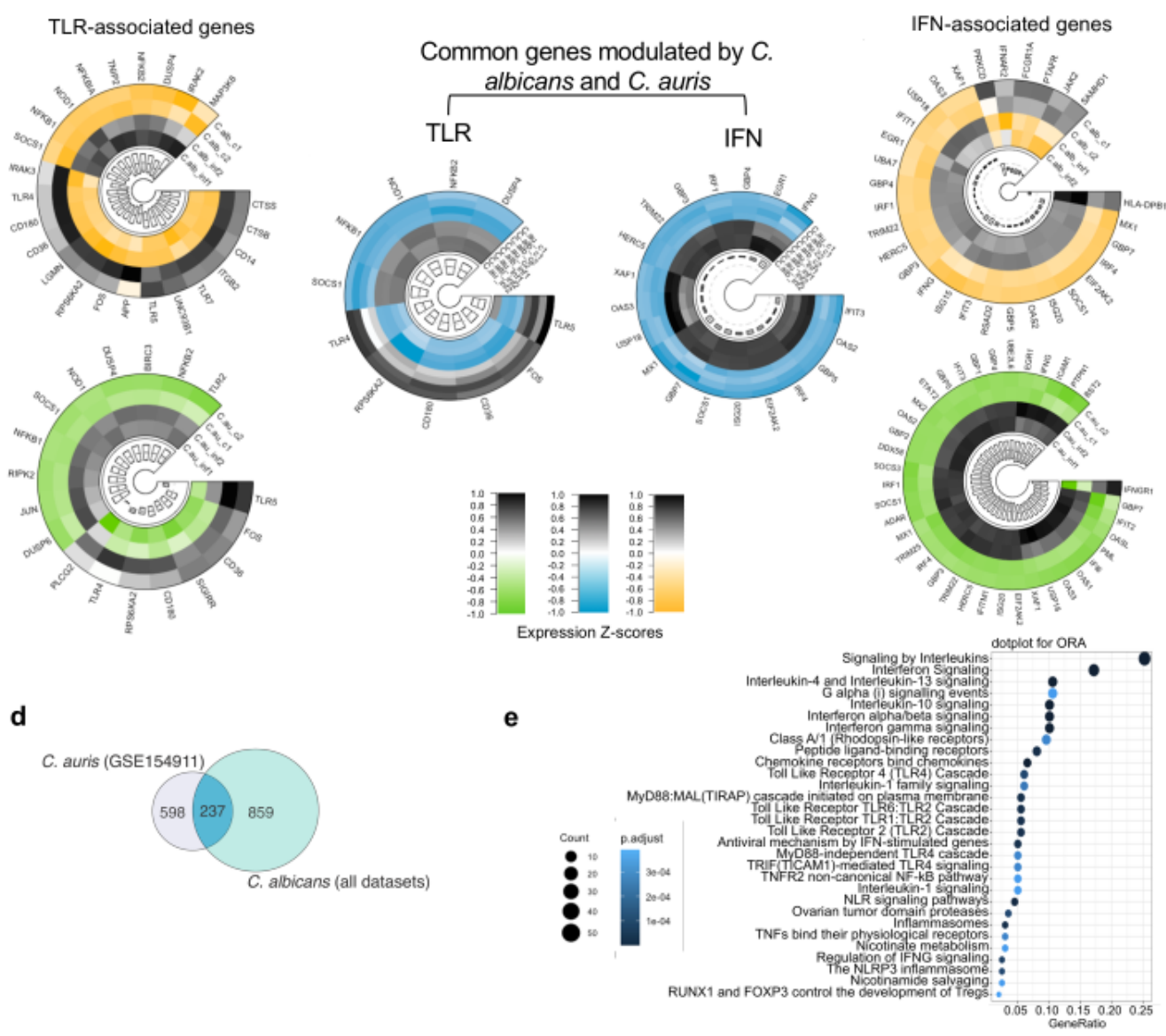

\section{Figure 5}

Induction of the interplay between TLR and IFN signaling pathways by other Candida species. Venn diagram showing the transcriptional overlap between $a$, TLR- and b, IFN-associated DEGs as well as the signaling pathways enriched in response to non-albicans candida species (C. glabrata, C. parapsilosis, and C. tropicalis) in comparison to C. albicans (created using CorelDraw2019, available at coreldraw.com). c, Circular heatmaps of RNAseq expression z-scores computed for log2 transformed 
DEGs ( $p$-value adj $<0.05$, fold change $>1$ and $<-1$ ) compares the expression of TLR- (left panels) and IFN(right panels) signaling pathways induced by C. albicans (green/grey heatmaps) or C. auris (yellow/grey heatmaps) all from GSE154911. Small circular heatmaps (blue/grey) demonstrate common DEGs modulated by $\mathrm{C}$. abicans and $\mathrm{C}$ auris. Individuals circular heatmaps were created using $\mathrm{R}$ packages circlize and ComplexHeatmap, while figure layout was edited using CorelDraw2019. d, Venn diagram showing the transcriptional overlap (an intersection containing 237 shared DEGs) induced by C. auris and C. albicans (those 1096 genes found across all studies: Suppl. Table S11). e, Dotplot of enriched signaling pathways by the 237 shared DEGs. DEGs, Differentially Expressed Genes; IFN, Interferon; ORA, Over representation analysis; TLR, Toll-like Receptor. 
Fig. 6

a

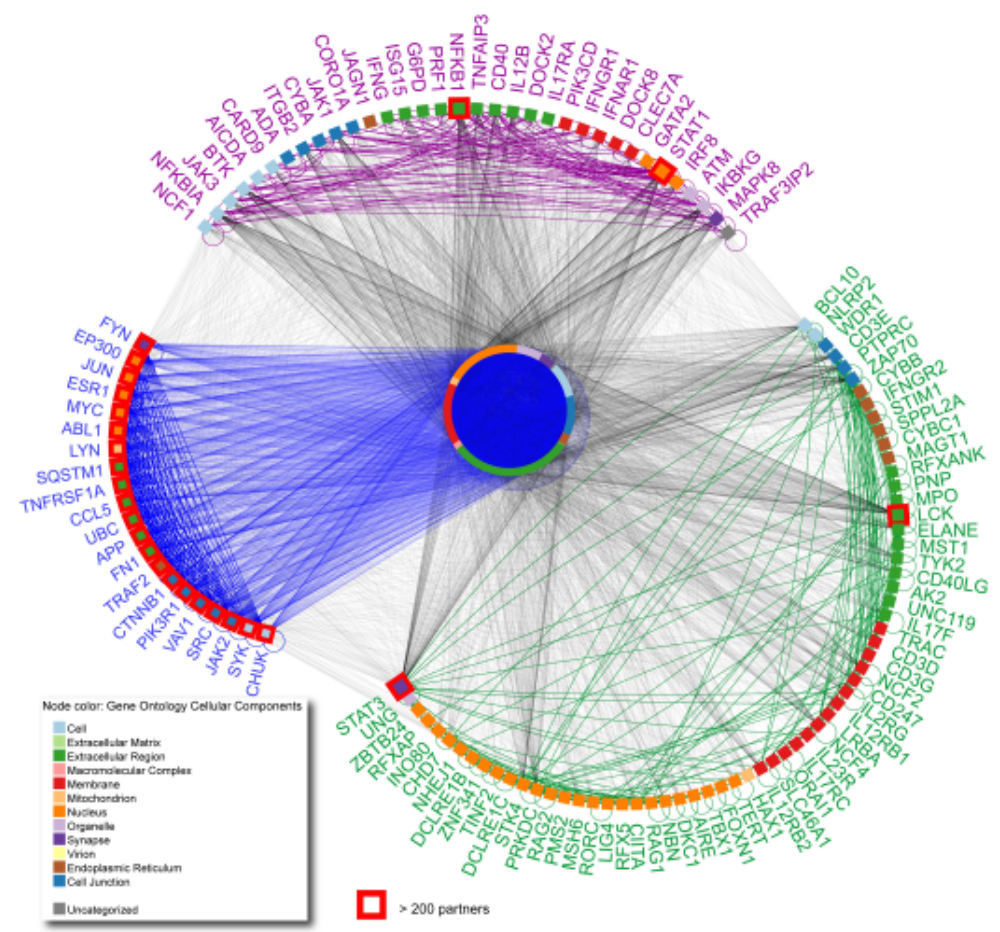

b

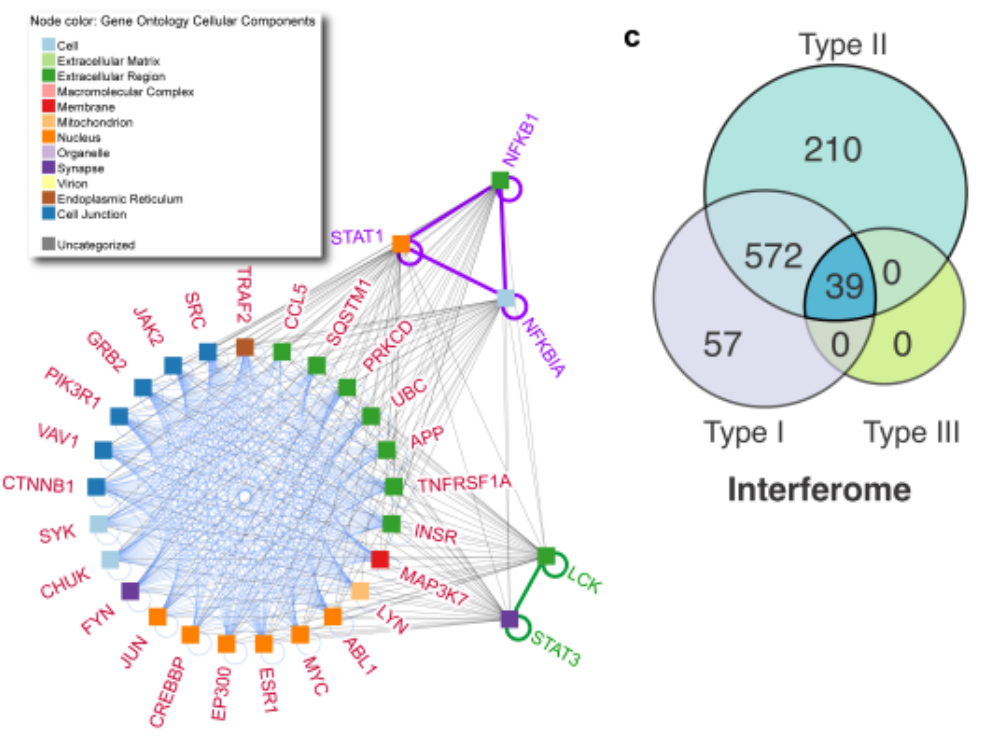

\section{Figure 6}

The interactome of DEGs enriching signaling pathways involved in the anti-candida immune response and its association with inborn errors of immunity. a, Relationships (edges) among the 1096 DEGs (nodes) found across all studies (Suppl. Table S11). Subnetworks (semicircles) represent genes associated with IEl causing increased susceptibility to candidiasis, being 34 purple nodes genes shared with the group of 1096 DEGs, while 66 green nodes represent those not found in the Candida datasets. 
Colored squares and circles represent the cell location of genes. The interaction network was build using the NAViGaTOR software. b, Network of hubs present in a. c, Proportional venn diagram (created using CorelDraw2019, available at coreldraw.com) of interferon types associated with the group of 1096 DEGs. Interferome analysis revealed 868 IFN-regulated genes modulated either by IFN type I, II, and III, as in the Venn Diagram. DEGs, Differentially Expressed Genes; IFN, Interferon; IEI, Inborn Errors of Immunity; TLR, Toll-like Receptor.

Fig. 7

a
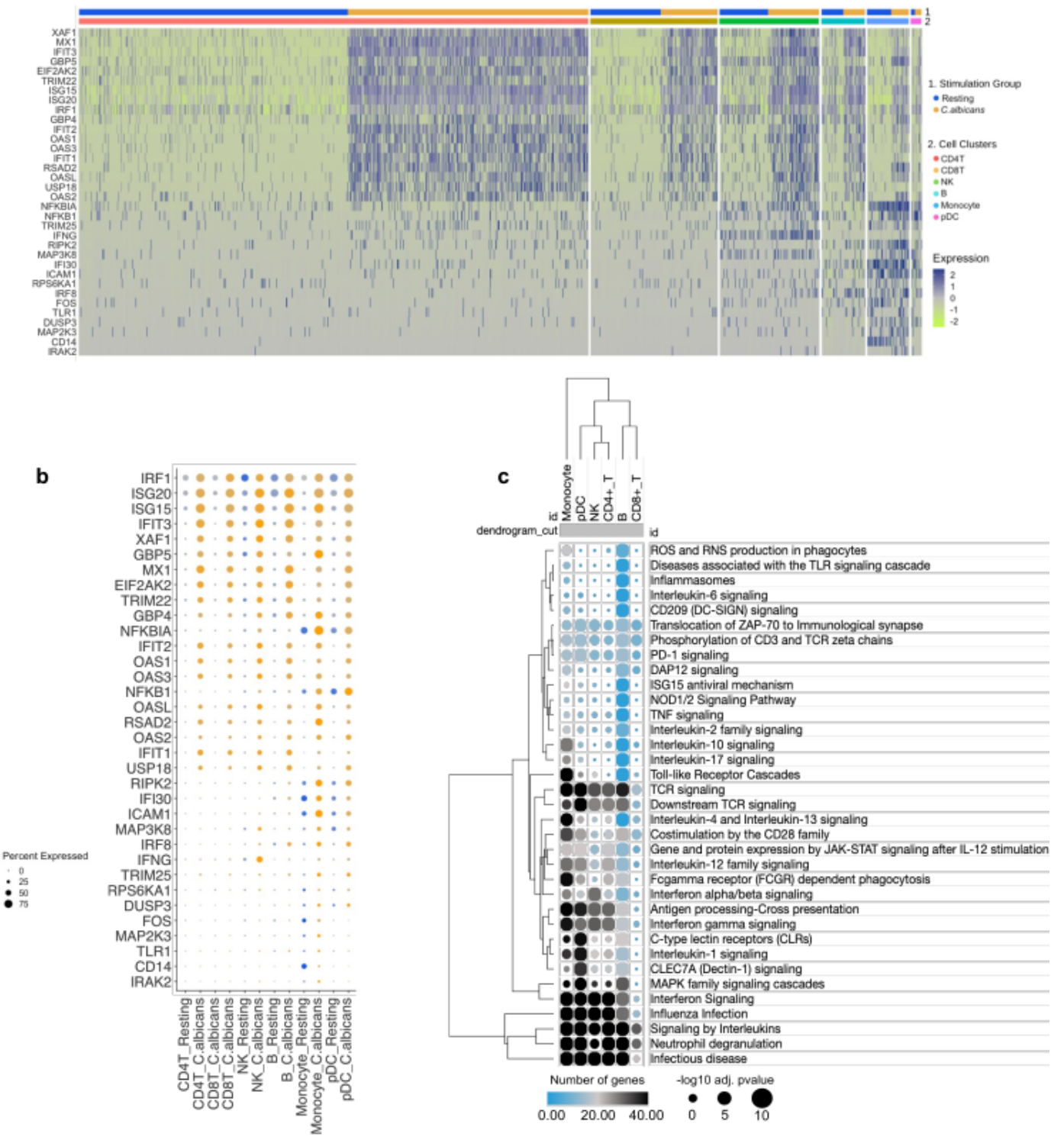
Common TLR- and IFN-associated DEGs and signaling pathways across microarray, bulk, and single-cell RNA-seq datasets. a, Heatmap using expression value from scRNAseq of DEGs also present in microarray and bulk studies; cell condition and group are indicated by different colors. b, Hierarchical clustering of average expression comparing resting and $\mathrm{C}$. albicans-activated cells. c, Hierarchical clustering showing common pathways selected from Fig. 1d, across the cell groups; the size of circles corresponds to adjusted p-value transformed into -Log10 and color intensity indicates the number of genes in each pathway across the cell groups, respectively. DEGs, Differentially Expressed Genes; IFN, Interferon; TLR, Toll-like Receptor; scRNAseq, single-cell RNA sequencing. 
Fig. 8

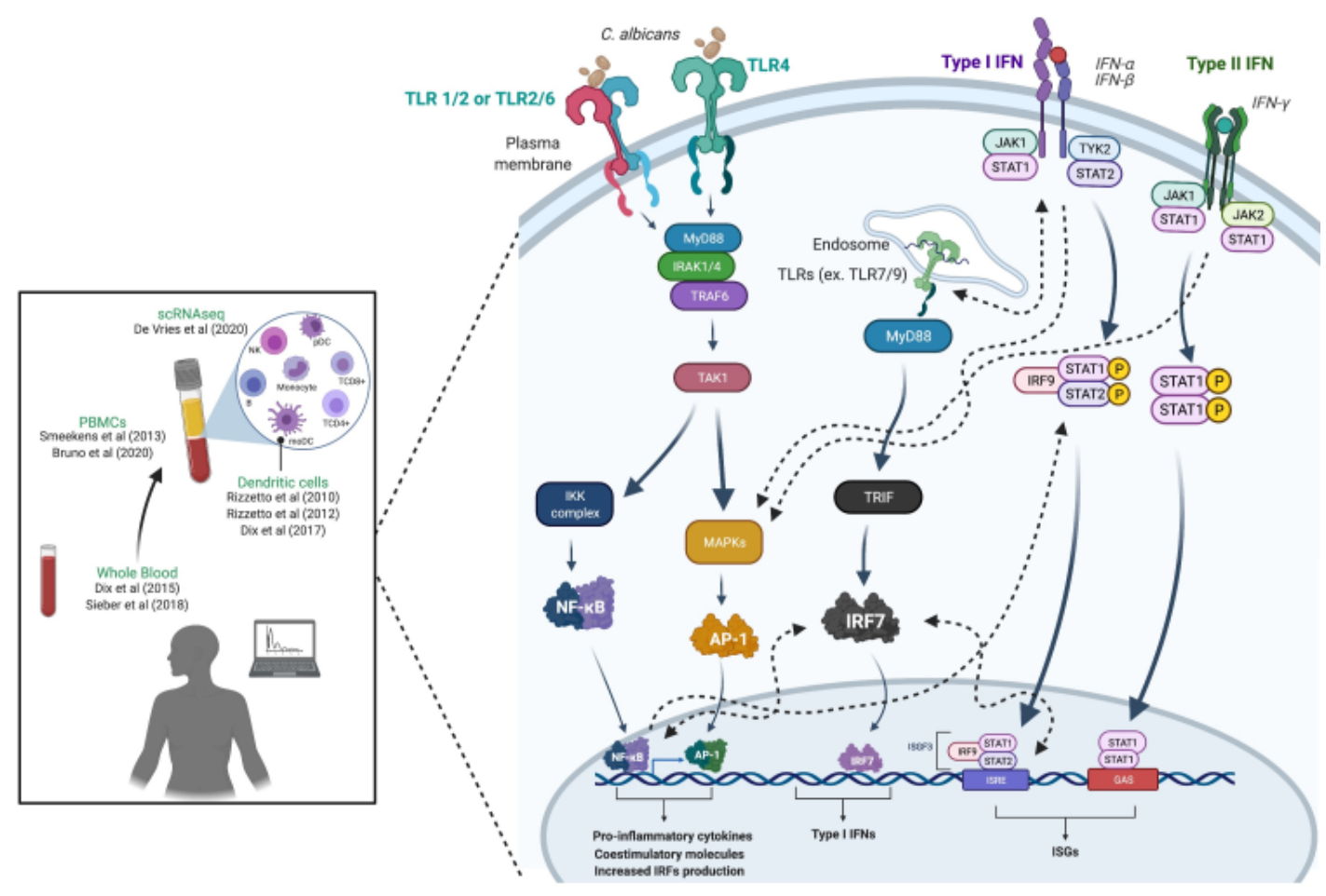

\section{Figure 8}

Schematic view summarizing studies and the interplay between TLR and IFN signaling pathways in the immune response to C. albicans. Pathways shown are reported in the literature50,103-109 (created using BioRender.com). IFN, Interferon; TLR, Toll-like Receptor.

\section{Supplementary Files}


This is a list of supplementary files associated with this preprint. Click to download.

- revisedSupplementaryTablesS1S18.xIsx

- revisedsupplementaryfigures.pdf 